\section{Office-based optical coherence tomographic imaging of human vocal cords}

\author{
Shuguang Guo, ${ }^{a}$ River Hutchison, ${ }^{b}$ Ryan P. Jackson, \\ Anu Kohli, ${ }^{\text {b }}$ Tristan Sharp, ${ }^{\text {b }}$ Elizabeth Orwin, \\ Richard Haskell, ${ }^{c}$ Zhongping Chen, ${ }^{\mathrm{a}, *}$ and \\ Brian J. F. Wong ${ }^{d, *}$ \\ aniversity of California, Irvine \\ Department of Biomedical Engineering \\ and \\ Beckman Laser Institute \\ Irvine, California 92697 \\ ${ }^{\mathrm{b}}$ University of California, Irvine \\ Beckman Laser Institute \\ Irvine, California 92617 \\ ${ }^{\mathrm{c}}$ Harvey Mudd College \\ Department of Physics \\ Claremont, California 91711 \\ ${ }^{\mathrm{d}}$ University of California, Irvine \\ Department of Biomedical Engineering \\ Beckman Laser Institute \\ and \\ Department of Otolaryngology-Head and Neck Surgery \\ Irvine, California 92697
}

\begin{abstract}
Optical coherence tomography (OCT) is an evolving noninvasive imaging modality and has been used to image the larynx during surgical endoscopy. The design of an OCT sampling device capable of capturing images of the human larynx during a typical office based laryngoscopy examination is discussed. Both patient's and physician's movements were addressed. In vivo OCT imaging of the human larynx is demonstrated. Though the long focal length limits the lateral resolution of the image, the basement membrane can still be readily distinguished. Office-based OCT has the potential to guide surgical biopsies, direct therapy, and monitor disease. This is a promising imaging modality to study the larynx. ๑ 2006 Society of Photo-Optical Instrumentation Engineers. [DOI: 10.1117/1.2200371]
\end{abstract}

Keywords: optical coherence tomography; vocal cords; biomedical imaging; early diagnosis; laryngeal cancer.

Paper 05375LR received Dec. 12, 2005; revised manuscript received Mar. 21, 2006; accepted for publication Apr. 5, 2006; published online May 11, 2006.

Early laryngeal cancer mimics benign disorders of the larynx, since both are characterized by identical symptoms such as throat pain, coughing, or hoarseness. Likewise, on physical examination it is difficult to distinguish early laryngeal cancer from morphologically similar benign disorders such as chronic laryngitis, even with the assistance of office-based flexible or rigid endoscopes. Both conventional examination and endoscopy lack the ability to visualize the depth of penetration of disease into the deeper layers of the larynx. Such

*Tel.: 949-824-6996; E-mail: bjwong@uci.edu or z2chen@uci.edu penetration through the layered structure of the vocal cord is the hallmark of invasive cancers.

As with most cancers, early diagnosis is essential for improved survival to prevent the spread within the organ or to other distant sites. Laryngeal cancer can only be diagnosed with a biopsy, but performing a biopsy on the vocal cord requires general anesthesia and surgical endoscopy. Further, biopsy of the vocal cord tissue itself is not without risk with the major complication being a potentially profound change in the voice quality, let alone the risks and costs associated with surgery, anesthesia, and patient time away from work. Delay and deliberation are common as the risks of a biopsy with surgical endoscopy may be considerable. Hence, there is a need to develop technology to assist with making the considerable decision to pursue surgery, guide and direct biopsies, and potentially diagnose early laryngeal cancer.

Optical coherence tomography (OCT) is an evolving noninvasive imaging modality that combines interferometry with low-coherence light to produce high-resolution images in turbid media such as living tissues, ${ }^{1}$ and has been used to image the larynx during surgical endoscopy. ${ }^{2-4}$ OCT imaging of the larynx in awake patients in an office setting has been limited because several challenges exist including patient and physician movements and the position of the larynx deep within the neck. In this paper, we discuss the design and use of an OCT sampling device capable of capturing in vivo images during awake office-based rigid laryngeal endoscopy.

A rigid laryngeal endoscope allows visualization of the larynx at an angle of either 70 or $90 \mathrm{deg}$ when placed into the oral cavity [Fig. 1(a)]. The tip of the laryngoscope is inserted into the oropharynx and is typically positioned a few centimeters above the vocal cords. These endoscopes provide the best images of the vocal cords short of performing endoscopy under general anesthesia. For our study, the laryngoscope serves as a platform to which a second device can be attached to perform simultaneous OCT imaging. The plane of section for imaging can be either in the transverse or sagittal plane of larynx; in this study the transverse plane was selected as it is the lateral (to the left or right) extension of diseases processes with which clinicians are most concerned.

There are several of factors that must be considered in the OCT laryngeal device design. The device should be able to be used in tandem with a conventional laryngoscope. It is potentially dangerous to touch the larynx when the patient is awake as this may trigger laryngospasm, which precipitates immediate airway closure and asphyxiation. Optimally, the imaging apparatus must be cantilevered in air within the pharynx. This requires long focal length optics, and this factor combined with the small vertical real estate in the oral cavity results in small numerical apertures. The result is a reduction in signal intensity and reduced lateral image resolution relative to more commonly used endoscopic OCT systems. ${ }^{2}$ The intensity of the signal is also affected by the angle of incidence the beam makes with the tissue.

An OCT system has two arms, the reference arm and sample arm. OCT depth scanning is performed by a rapid scanning optical delay line in the reference arm. ${ }^{5}$ In the sample arm, only the backscattered signal of which the optical path length matches that of the reference arm can be detected.

1083-3668/2006/11(3)/030501/3/\$22.00 @ 2006 SPIE 


\section{JBO LETTERS}

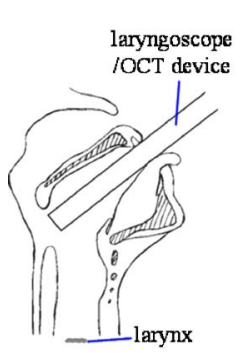

(a)

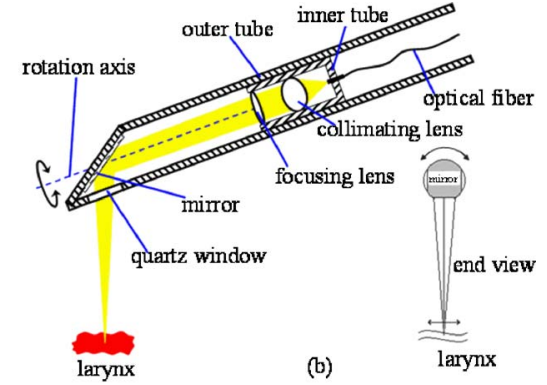

(b)

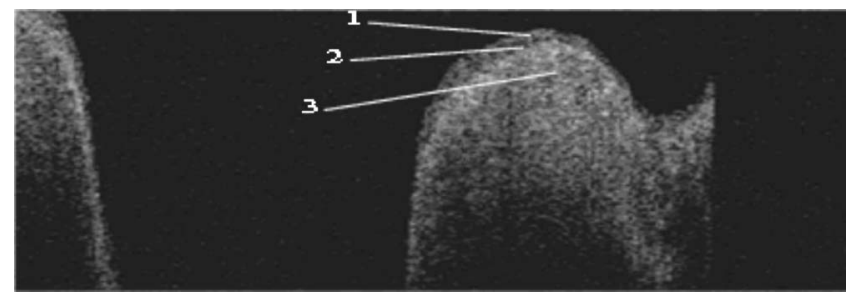

Fig. 3 In vivo OCT image of healthy vocal cords. The basement membrane is clearly visible: 1, epithelium; 2, basement membrane; 3, lamina propria.

sketch of the laryngeal OCT sampling probe.

The majority of OCT probes developed for endoscopic applications have fixed optical arrangements, and thus a fixed working distance. In laryngeal endoscopy, the depth of the larynx in the throat and the path length light must travel from the incisors to the vocal cords vary markedly from patient to patient. Hence, a mechanism is required to allow active adjustment of the working distance. However, changing the optical path length of the reference arm to match a variable working distance is difficult for several reasons, the most important of which is that there is no means of knowing a priori whether the sampling beam is optimally focused on the specimen. Secondly, the depth of the larynx in the throat changes with patient posture and position, dynamically. The most convenient solution is to maintain a constant optical delay in the sample arm during tuning of the working distance to ensure that the depth scanning range is always in focus, since the device must quickly adjust to image the larynx as it changes position within the pharynx. A depth adjustment function is essential for tracking the larynx (see below).

The scanning mechanism consists of two concentric hollow cylinders [Fig. 1(b)]. The sample beam from the OCT system is collimated, passes through a focusing lens, and is reflected down to the larynx. The fiber and collimating and focusing lenses are also housed in an innermost tube that can be moved back and forth by a motor to adjust the working distance over a range of $4 \mathrm{~cm}$, allowing for the focus point to be 4 to $8 \mathrm{~cm}$ below the probe tip. The outer tube has an angled mirror at its terminus, which deflects the sampling beam at a 70-deg angle to reach the larynx. This outer tube is free to rotate about $10 \mathrm{deg}$ and is coupled to a gearhead and servocontrolled motor (Model 1724T024SR, MicroMo Elec-

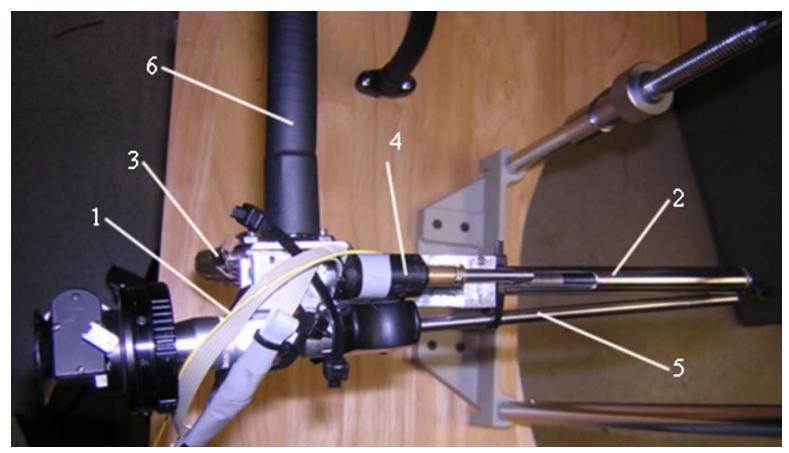

Fig. 2 Picture of the OCT sampling probe attached to the laryngoscope: 1, sampling fiber; 2, two coaxial tubes; 3, scanning motor; 4, depth-tracking motor; 5, laryngoscope; 6, gooseneck. tronics, Clearwater, Florida) to achieve lateral scanning. Since the position of lenses relative to the fiber is fixed, the optical delay of the focal point remains constant during adjustment of the working distance. Hence one optical path length fits all variable working distances.

A glass tube with a flat optical window at its terminus encloses both cylinders. It serves to shield the outer and innermost tubes from fluids within the oral cavity and pharynx and, since it can be sterilized, ensures that each patient is examined with a clean instrument. Finally, the device is coupled to the laryngoscope by a carriage made of Delrin. The endoscope and the OCT device are held together in a "doublebarreled" configuration; the carriage in turn can be secured to a flexible gooseneck joint, which supports the weight of the device and associates video capture equipment (for the endoscope) (Fig. 2).

The greatest challenge posed by transoral awake officebased OCT imaging is the amount of patient movement encountered during examination. This is due to natural movements of the head and neck and involuntary movements of the larynx produced by swallowing, breathing, and native reflexes. There are also significant tremors in the physician's hand when he or she positions the instrument and this is magnified by the cantilevered nature of the instrumentation. Since the image acquired is only a few square millimeters, the small movement and tremor of both the physician's hand holding the device and the patient's head, neck, larynx, and torso produces severe motion artifacts.

To reduce the impact of these effects, we employed a forehead rest that restrains the external motion of the patient's head yet allows for quick withdrawal should a gag reflex be triggered or if the patient experiences discomfort. An ophthalmic slit lamp stand was modified to provide a frame for the forehead rest and the chin rest was removed as the mandible must freely open during examination and cannot be constrained (Fig. 2). A gooseneck clamped to the device and mounted on a table dampens the physician's hand while allowing control over the position of the device in the patient's mouth.

The core OCT device has been described previously. ${ }^{6,7}$ The system used in this study employed a low-coherence light source (central wavelength $\lambda=1310 \mathrm{~nm}$, AFC Technologies, Hull, Quebec, Canada). An aiming beam (wavelength $635 \mathrm{~nm}$ ) is combined with the OCT sampling arm so that the scanning region can be identified by the otolaryngologist when the image is viewed with a conventional laryngoscope. Images are acquired at a frame rate of $1 \mathrm{~Hz}$.

Five subjects were evaluated under aegis of the Institutional Review Board at the University of California, Irvine. 
Images of the larynx were obtained in five objects. Figure 3 shows a representative acquired OCT image. The epithelium, the basement membrane, and the lamina propria can be clearly identified. The depth resolution of the image is $7 \mu \mathrm{m}$ and the lateral resolution is $20 \mu \mathrm{m}$. The image size is $1.6-\mathrm{mm}$ depth by $5.2-\mathrm{mm}$ width. There is some motion artifact present. The image is comparable with images obtained in anesthetized patients during surgical endoscopy in which the demarcation between the lamina propria and basement membrane can be identified. ${ }^{2}$ It differs in terms of image resolution and the obvious motion artifact.

The present device requires a minor paradigm shift in the way the larynx is examined. In conventional transoral rigid laryngeal endoscopy, the otolaryngologist holds the endoscope in his hand and positions the tip of the device in the oropharynx. Because the large field of view is typically at least $3 \times 3 \mathrm{~cm}$ in size and the endoscopes have a relatively large depth of focus, movement of the instrument (or surgeon's hand) or the patient is tolerated. In awake laryngeal OCT imaging, a relatively small region of space is interrogated. Shifts in the position of even $1 \mathrm{~mm}$ can cause the target site to exit the object plane of the OCT device. Hence, it is imperative that these movements be constrained. The use of a bracket system to reduce head and torso position as well as a gooseneck stabilizer for the endoscope is a novel adaptation. Harnesses to reduce motion are commonly used in other medical specialties such as ophthalmology where even more invasive procedures are performed such as retinal vessel photocoagulation.

There are only three ways in which an otolaryngologist can visual the larynx and vocal cords in an office-based setting. The most common though least reliable technique involves the use of a dental mirror, which is placed in the pharynx. This is a good screening technique, but provides no means for image capture and archival. Fiber optic and rigid endoscopes are used as well via transnasal and transoral placement. The flexible fiberoptic laryngeal endoscope is inserted through the nose and is suspended above the larynx and can be positioned in near approximation to the cords. However, it is difficult to incorporate a complex OCT lateral scanning mechanism, and hence only simple raster scans produced by the back-andforth translation of the fiber can be accomplished. Images obtained using this platform only provide information on the free margin of the vocal cord, which is of less clinical value to physicians. The other platform is the rigid laryngeal telescope, which we have adopted in this study.

Alternative optical designs of the laryngeal OCT probe are possible but either difficult to operate or very complex. A probe with a fixed focus and a constant working distance is probably more stable. However, the otolaryngologist has to track the focus by shifting the whole probe up or down, which is difficult due to the limited space of the oropharynx. As to designs with a variable working distance, conventional dynamic focusing systems have no consideration of the change in optical path length while an OCT probe does. In our design, the relative position of lenses to the fiber is fixed, thus the optical delay of the focal point is invariable while changing the working distance. Therefore, the delay line of the reference arm can be fixed, which results in a simple and efficacious design.
A major technical challenge we encountered is image localization, which is very important as the larynx can move with 6 degrees of freedom and superior-inferior motion can shift the target surface out of the focal region of the device. The use of a second motor system that maintains the optical delay but varies the working distance largely solved this problem (in tandem with the use of a harnessing system to stabilize the head of the subject), but still the present system is limited in that the scan is too slow. Images are acquired at a frame rate of $1 \mathrm{~Hz}$, but the motion of the larynx and intention tremor of the surgeons' hand can exceed that, which makes finding the target surface challenging at times. Obviously, using a high-speed OCT system would be the solution. Currently we are building such a system, but because the lenses, inner tube, and mirror collectively form a device with a relatively large moment of inertia, the dc motors and gearheads cannot move reliably beyond 1 to $2 \mathrm{~Hz}$. Alternate optomechanical designs will be needed for a high-speed system, and this is currently an active project in our laboratory.

In summary, we demonstrate an OCT sampling device capable of capturing in vivo images during a typical office-based laryngoscopy examination. Both the patient's and physician's movements were addressed. Though the long focal length limits the lateral resolution of the image, the basement membrane can still be readily distinguished. Office-based OCT has the potential to guide surgical biopsies, direct therapy, and monitor disease. This is a promising imaging modality to study the larynx.

\section{Acknowledgments}

Rachel Lovec, Tonya Icenogle, Senecca Harberger, and Nikhil Gheewala from Harvey Mudd College participated in the design and construction of the device. This work was supported by the National Institutes of Health (DC 006026, CA 91717, EB 00293, RR 01192, RR00827), National Science Foundation (BES-86924), Flight Attendant Medical Research Institute (32456), State of California Tobacco Related Disease Research Program (12RT-0113), Air Force Office of Scientific Research (F49620-00-1-0371), and the Arnold and Mabel Beckman Foundation. Jiangping Su, Jorge Perez, David Vokes, and William Armstrong assisted with the evaluation of the device.

\section{References}

1. D. Huang, E. A. Swanson, C. P. Lin et al., "Optical coherence tomography," Science 254, 1178-1183 (1991).

2. B. J. F. Wong, R. P. Jackson, S. Guo et al., "In vivo optical coherence tomography of the human larynx: normative and benign pathology in 82 patients," Laryngoscope 115, 1904-1911 (2005).

3. A. M. Sergeev, V. M. Gelikonov, G. V. Gelikonov et al., "In vivo endoscopic OCT imaging of precancer and cancer states of human mucosa," Opt. Express 1, 432-440 (1997).

4. A. V. Shakhov, A. B. Terentjeva, V. A. Kamensky et al., "Optical coherence tomography monitoring for laser surgery of laryngeal carcinoma," J. Surg. Oncol. 77, 253-258 (2001).

5. G. J. Tearney, B. E. Bouma, and J. G. Fujimoto, "High-speed phaseand group-delay scanning with a grating-based phase control delay line," Opt. Lett. 22, 1811-1813 (1997).

6. Y. Zhao, Z. Chen, C. Saxer et al., "Phase-resolved optical coherence tomography and optical Doppler tomography for imaging blood flow in human skin with fast scanning speed and high velocity sensitivity," Opt. Lett. 25, 114-116 (2000).

7. H. Ren, Z. Ding, Y. Zhao et al., "Phase-resolved functional optical coherence tomography: simultaneous imaging of in situ tissue structure, blood flow velocity, standard deviation, birefringence, and Stokes vectors in human skin," Opt. Lett. 27, 1702-1704 (2002). 